\title{
The radiating pressure field of a turbulent cylindrical cavity flow
}

\author{
M. Grottadaurea* and A. Rona ${ }^{\dagger}$ \\ University of Leicester, Leicester LE1 7RH, United Kingdom
}

\begin{abstract}
The unsteady flow past a cylindrical cavity at $M=0.235$ is investigated by Detached Eddy Simulation. Two different length to depth ratios $L / D=2.5$ and $L / D=0.713$ are studied to evaluate their influence on the flow. The time-resolved numerical method predicts a flow characterized by a large-scale periodic instability with a characteristic period $\mathcal{T}$ that changes with the depth. In the $L / D=2.5$ cavity, $\mathcal{T}=0.7745 L / u_{\infty}$ and the peak pressure fluctuation in the near-field $p^{\prime}=15 \mathrm{~Pa}$. The $L / D=0.713$ cavity is characterized by larger pressure fluctuations with a maximum of $p^{\prime}=40 \mathrm{~Pa}$ and a longer fundamental instability period $\mathcal{T}=1.853 L / u_{\infty}$. The directivity of the radiating near-field pressure fluctuation differs between the two configurations. These have a similar upstream main directivity peak but the $L / D=0.713$ cavity displays also a secondary downstream peak and it is about $15 \mathrm{~dB}$ louder. In the spanwise plane, the acoustic wave from the $L / D=2.5$ cavity is asymmetric, whereas it is symmetric in the $L / D=0.713$ cavity. This characterization offers some scope for formulating airframe noise reduction strategies that are configuration specific to shallow and deep cylindrical cavities.
\end{abstract}

\section{Nomenclature}

c Speed of sound

$D \quad$ Cylindrical cavity depth

$d \quad$ Distance from the nearest wall

$\Delta \quad$ Filtering lenght, $\Delta=\sqrt[3]{\delta V}$

$e \quad$ Internal energy

$f \quad$ Characteristic frequency

$k \quad$ Turbulent kinetic energy

$\nabla \quad$ Gradient operator

$L \quad$ Cylindrical cavity diameter

$\mu_{l} \quad$ Dynamic viscosity

$M \quad$ Mach number

$\mu_{t} \quad$ Eddy viscosity

n Unit inward normal vector

$\nu \quad$ Kinematic viscosity

$\omega \quad$ Specific dissipation rate of turbulent kinetic energy

$p \quad$ Pressure

$\operatorname{Pr} \quad$ Prandtl number, $\operatorname{Pr}=0.72$

$\mathrm{Pr}_{t} \quad$ Turbulent Prandtl number, $\mathrm{Pr}_{t}=0.9$

$\mathbf{q} \quad$ Heat flux vector, $\mathbf{q}=\frac{1}{\gamma-1}\left(\frac{\mu}{P r}+\frac{\mu_{t}}{P r_{t}}\right) \nabla T$

$R e_{x}$ Reynolds number based on a generic length $x, R e_{x}=\frac{u_{\infty} x}{\nu}$

$\rho \quad$ Density

$S P L$ Sound Pressure Level

St Strouhal number, $S t=f L / u_{\infty}$

\footnotetext{
${ }^{*} \mathrm{PhD}$ Student, Department of Engineering.

${ }^{\dagger}$ Lecturer, Department of Engineering, AIAA Member.
} 


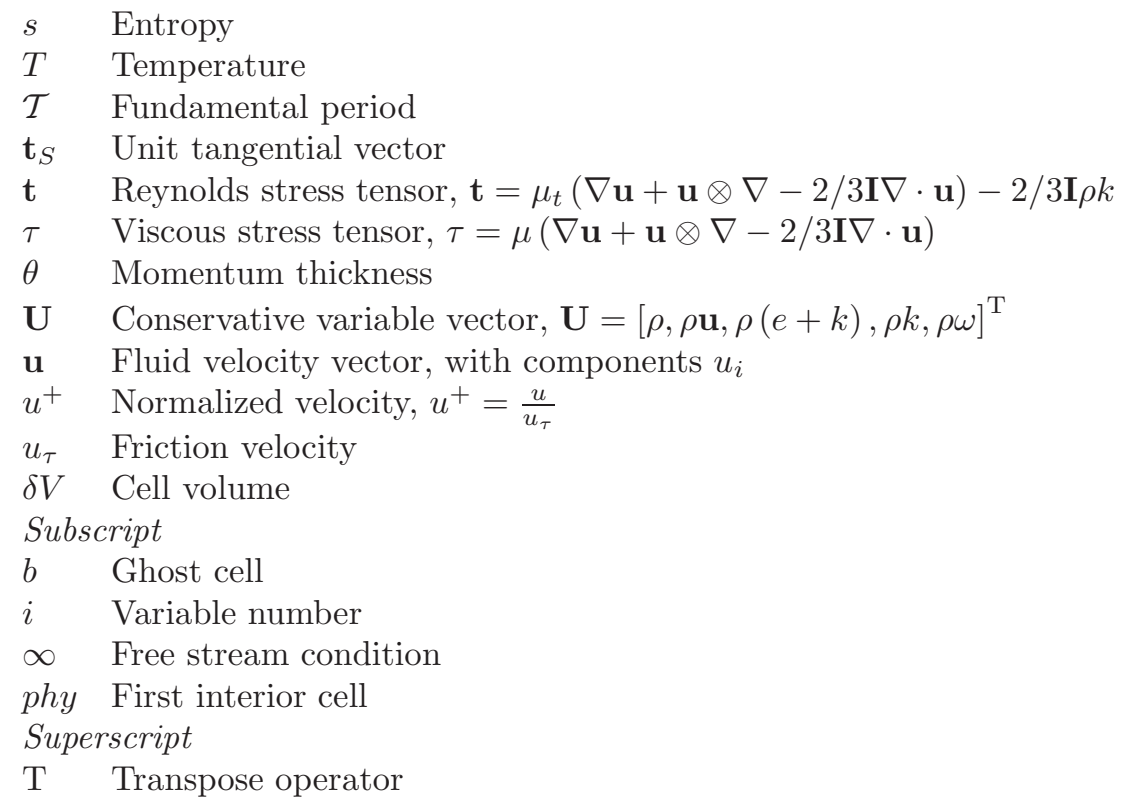

\section{Introduction}

Recesses or grooves often feature in a civil aircraft to accommodate service hatches and other ancillary equipment. The flow in these cavity-shaped recesses is unsteady and, at typical landing speeds, may features large-scale instabilities. In a civil aircraft, the high lift systems and the landing gear are the most acoustically active airframe components. Nonetheless, other components, such as fuel vents or ailerons, also contribute to the overall noise emissions.

A cylindrical open cavity placed one metre downstream of a flat plate leading edge is herein investigated as an initial low fidelity model of a fuel vent widebody civil aircraft at landing. At typical landing speeds, the flow is characterized by a large Reynolds number and a low Mach number. To study the flow unsteadiness of such a cavity, a compressible Detached Eddy Simulation in-house flow solver is used, which is a hybrid between the RANS $k-\omega$ model of Wilcox $^{1}$ and the Yoshizawa ${ }^{2}$ one-equation LES model. The curvature of the cavity walls adds to the modelling effort. Past cavity aeroacoustic investigations mainly focussed on rectangular enclosures, due to the savings in computer time that can be achieved by the use of a Cartesian mesh. The computational resources now available enable the use of a curvilinear conformal mesh to tackle a more complex geometry such as that of a cylindrical cavity. ${ }^{3,4}$

The experimental work by Dybenko and Hering ${ }^{4,5}$ and the numerical work by Grottadaurea and Rona ${ }^{6,7}$ show that cylindrical cavities develop a rather unique flow instability that is fundamentally different from the well-documented Rossiter mechanism. ${ }^{8}$ The presence of cylindrical cavity geometries on airframes motivates the investigation and qualification of this peculiar instability mechanism. Symmetric and anti-symmetric modes can be encountered in this geometry ${ }^{9}$ and the mode selection and mode switching are yet to be investigated.

From the available literature, ${ }^{10}$ it is known that the momentum thickness $\theta$ at the cavity leading edge plays an important role in the selection of the modes and in governing the growth of the shear layer ${ }^{10-12}$ that $^{1}$ spans an open cavity. ${ }^{13}$ Given the known importance of $\theta$ on the mode selection of rectangular cavities, ${ }^{14}$ this paper aims to include the modelling of this parameter in selected cylindrical cavities, to capture the main cavity flow instability mechanism and its amplitude.

The radiating pressure fluctuation away from the cavity opening is studied by extracting the near-field unsteady pressure from the numerical predictions. This gives an entry level survey of the radiation pattern that is expected to characterize the acoustic far-field, which is relevant to airframe noise applications. The objective is to relate the flow structures that are modelled inside and around the enclosure to the near-field sound directivity. In particular, it is of interest to explore whether the asymmetry in the cylindrical cavity flow instability that was predicted in past numerical work ${ }^{6,7}$ results in an asymmetric radiation of sound from these enclosures. 


\section{Test-case geometry and inflow conditions}

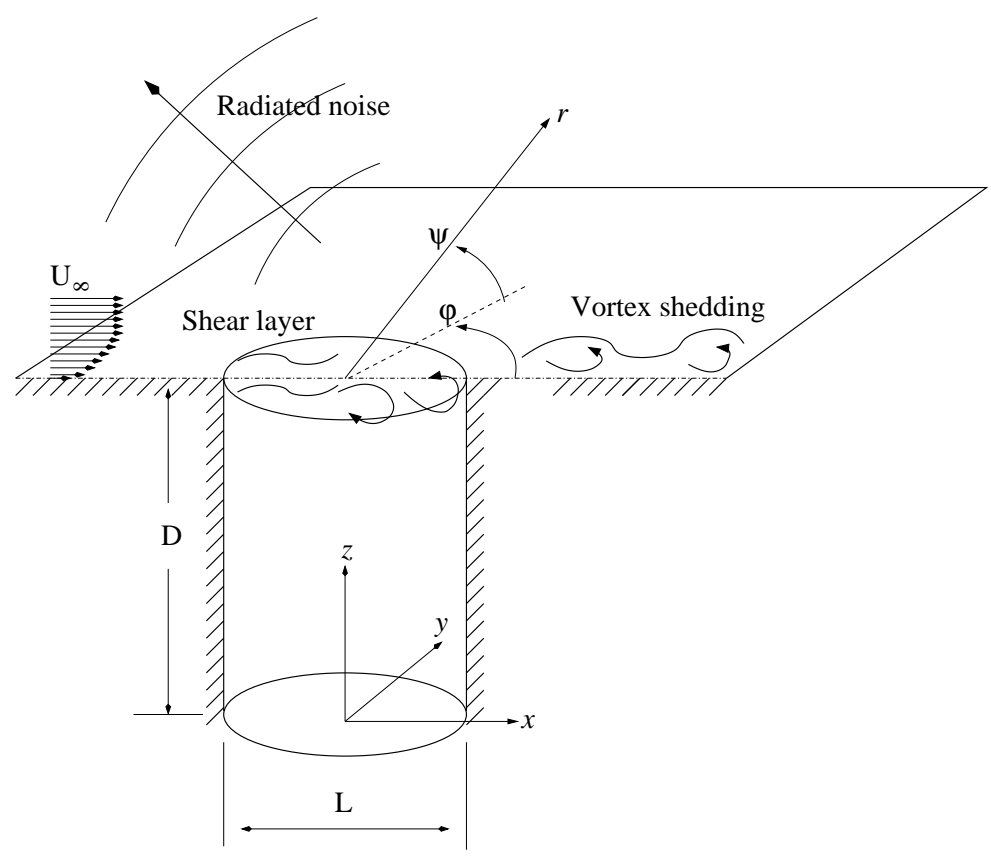

Figure 1. Cylindrical cavity flow.

Figure 1 shows a simplified sketch of the cavity flow in the cartesian coordinates $(x, y, z)$. An auxiliary reference system in polar coordinates $(\psi, \phi, z)$ is used in section IV to discuss the acoustic predictions. The cartesian coordinate system $(x, y, z)$ origin is centred at the bottom of the cavity. The origin of the polar reference system $(\psi, \phi, r)$ is the centre of the cavity open end.

The following dimensional variables characterize the cavity flow: ${ }^{15} L, D, u_{\infty}, \theta, c_{\infty}$ and $\nu_{\infty}$. These can be rearranged into the corresponding non-dimensional parameters: $L / D, L / \theta, \operatorname{Re}_{\theta}, M_{\infty}$.

A cylindrical cavity placed one meter downstream of a flat plate is simulated. This study considers two specific variants of this basic configuration. Two cylindrical cavities of diameter $(L)$ of $100 \mathrm{~mm}$ and depths $(D)$ of $40 \mathrm{~mm}$ and $140 \mathrm{~mm}$ are studied at $u_{\infty}=80 \mathrm{~m} / \mathrm{s}$ at $\theta=1.54 \mathrm{~mm}$. The flow is at International Standard Atmospheric (ISA) ground conditions, which gives $c_{\infty}=340.3 \mathrm{~m} / \mathrm{s}$ and $\nu_{\infty}=1.461 \times 10^{-5} \mathrm{~m}^{2} / \mathrm{s}$.

Rearranging the previous dimensional parameters, the following non-dimensional parameters are considered in the simulation: $L / D=2.5,0.713, L / \theta=65, R e_{\theta}=8760$ and $M_{\infty}=0.271$. The Reynolds numbers based on distance along the flat plate $(x)$ upstream of the cavity leading edge is $R e_{x}=5.47 \times 10^{6}$.

\section{Numerical model}

\section{III.A. Governing equations}

The compressible turbulent flow is governed by the short-time averaged Navier-Stokes equations written in the conservative form

$$
\frac{\partial}{\partial t} \mathbf{U}+\nabla \cdot\left(\mathbf{F}_{c}+\mathbf{F}_{t}\right)+\mathbf{S}=0
$$

where the conservative vector $\mathbf{U}$, the convective flux vector $\mathbf{F}_{c}$, the turbulent flux vector $\mathbf{F}_{t}$ and the turbulent source terms vector $\mathbf{S}$ are given by ${ }^{16}$ 


$$
\begin{aligned}
\mathbf{U} & =[\rho, \rho \mathbf{u}, \rho(e+k), \rho k, \rho \omega]^{\mathrm{T}} \\
\mathbf{F}_{c} & =[\rho \mathbf{u}, \rho \mathbf{u} \otimes \mathbf{u}+p \mathbf{I}, \rho \mathbf{u}(e+p / \rho+k), \rho \mathbf{u} k, \rho \mathbf{u} \omega]^{\mathrm{T}} \\
\mathbf{F}_{t} & =\left[0,-(\mathbf{t}+\tau),-\mathbf{q}-(\mathbf{t}+\tau) \cdot \mathbf{u}-\left(\mu_{l}+\sigma_{1} \mu_{t}\right) \nabla k,-\left(\mu_{l}+\sigma_{1} \mu_{t}\right) \nabla k,-\left(\mu_{l}+\sigma_{2} \mu_{t}\right) \nabla \omega\right]^{\mathrm{T}} \\
\mathbf{S} & =\left[0, \mathbf{0}, 0, \Gamma \beta_{1} \rho k \omega+(1-\Gamma) C_{d} \rho \frac{k^{3 / 2}}{\Delta}-\mathbf{t}: \nabla \mathbf{u}, \beta_{2} \rho \omega^{2}-\frac{\alpha \omega}{k} \mathbf{t}: \nabla \mathbf{u}\right]^{\mathrm{T}}
\end{aligned}
$$

In equations (4) and (5), the following algebraic relations are used:

$$
\begin{aligned}
\mu_{t} & =\Gamma \frac{k}{\omega}+(1-\Gamma) C_{s} \sqrt{k} \Delta \\
\Gamma & =\tanh \left(\eta^{4}\right) \\
\eta & =\max \left(\frac{\sqrt{k}}{0.09 \omega d}, \frac{500 \nu}{\omega d^{2}}\right)
\end{aligned}
$$

The switching between the Wilcox $k-\omega$ RANS turbulent closure model and the Yoshizawa one-equation LES model is achieved by the introduction of a blending function $\Gamma{ }^{17}$ In the near-wall region $(d \rightarrow 0), \Gamma$ approaches unity and the RANS model is predominant. Away from the wall $(d \gg 0), \Gamma$ tends to zero and the one-equation LES model is recovered. The turbulence model closure coefficients are given in table ??. ${ }^{1,2}$ The conservative variables $\mathbf{U}$ are computed by an explicit in-house finite volume flow solver that is up to second order accurate in space and time. ${ }^{18}$

\begin{tabular}{|c|c|c|c|c|c|c|}
\hline$\sigma_{1}$ & $\sigma_{2}$ & $\beta_{1}$ & $\beta_{2}$ & $\alpha$ & $C_{s}$ & $C_{d}$ \\
\hline $1 / 2$ & $1 / 2$ & $9 / 100$ & $9 / 125$ & $13 / 25$ & 0.07 & 1.05 \\
\hline
\end{tabular}

Table 1. Turbulence closure model coefficients.

\section{III.B. Computational mesh}

A conformal curvilinear mesh is used to model the cylindrical cavities defined in section II. The computational domain is shown in figure 2. By domain decomposition, the domain is split into 6 zones. The zones have a similar number of cells to even out the computational load among the processors in the MPI implementation of the code.

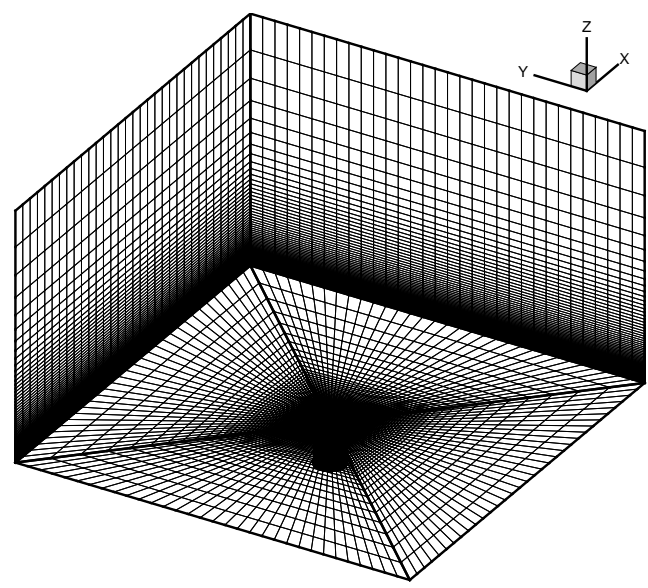

(a) Computational mesh

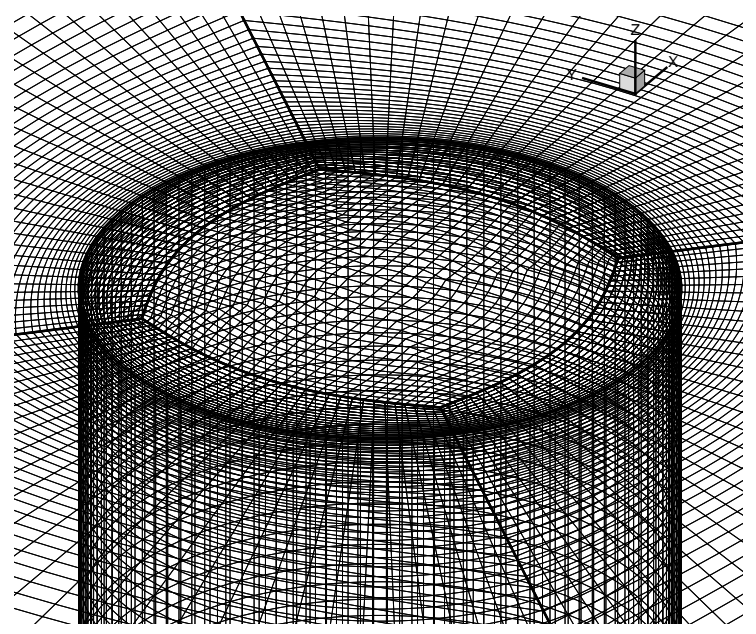

(b) Detail of the mesh in the enclosure

Figure 2. Computational domain details 
Previous numerical investigations of this cylindrical cavity configuration ${ }^{6,7}$ showed that a computational domain of size $13 L \times 13 L \times 9 L$ outside the cavity is required to resolve at least one full acoustic wavelength of the radiating sound. Therefore a domain of $13 L \times 13 L \times 9 L$ is used on this work.

1.3 million cells are used in the $L / D=0.71$ cavity model and 1.26 million cells in the $L / D=2.5$ model. 128 points are used around the cavity circumference. The mesh skewness over the cavity opening was investigated and minimized in Grottadaurea and Rona. ${ }^{7}$ An advantage of the generated computational mesh is the modest deformation of the cells around the cylinder walls. The first cell centre in the boundary layer correspond to $z^{+}=30$ and a wall function is used to impose the non-slip condition, as described in section III.C.

The mesh convergence of the $L / D=0.71$ predictions has been investigated by running a 2.6 million cells mesh refined test case. 256 equispaced points are used around the cavity circumference in this test case. The code was run over 72 processors at the supercomputer facilities HECToR and BCX. HECToR is a 11,328 AMD $2.8 \mathrm{GHz}$ Opteron processors cluster, delivering 59 Tflops at peak, located in Edinburgh, UK. BCX is a 2560 Opteron IBM Dual Core 2.6 GHz processors cluster, located in CINECA, Bologna, IT.

\section{III.C. Boundary conditions and connectivity}

To keep the second order accuracy of the flow solver at the boundaries of the computational domain, each zone is fully rinded using ghost cells, where the boundary flow state is imposed. A ghost cell is generated by mirroring the first interior cell about the boundary plane.

Dividing the computational domain in zones generates inter-block boundary cells. The latters are defined as the ghost cells corresponding to the physical cells of a neighboring zone. For a general connectivity between two zones $(1,2)$, the state in the inter-block boundary ghost cells is defined as $\mathbf{U}_{b, 1}=\mathbf{U}_{p h y, 2}$.

The Spalding law of the wall is used to estimate the tangential flow speed at the first interior point, with the von Kàrmàn constant $\kappa=0.41$ and $B=5.0 .^{19} k$ and $\omega$ are computed according to a compressible model by Wilcox. ${ }^{1}$ The ghost cell tangential velocity $\left(\mathbf{u}_{b} \cdot \mathbf{t}_{S}\right)$ is computed using equation 9 to give the correct friction velocity $u_{\tau}$ at the wall. In equation $9, z^{+}$is the non-dimensional wall-normal distance, $z^{+}=d u_{\tau} / \nu$.

$$
\begin{aligned}
\widetilde{z} & =e^{-\max \left[0,\left(\frac{z^{+}-5}{20}\right)\right]} \\
\widetilde{u} & =\widetilde{z}+\left(\frac{1-\widetilde{z}}{\kappa u^{+}}\right) \\
\mathbf{u}_{b} \cdot \mathbf{t}_{S} & =\mathbf{u}_{p h y} \cdot \mathbf{t}_{S}(1-2 \widetilde{u})
\end{aligned}
$$

The computational domain through-flow boundaries are modelled using the $3 \mathrm{D}$ extension ${ }^{6}$ of the $2 \mathrm{D}$ far-field non-reflective boundary condition of Manna, ${ }^{18}$ based on the work of Giles. ${ }^{20}$ As required by the CFD General Notation system, ${ }^{21}$ this is a switching condition between non-reflective inflow and outflow, depending on the flow-normal velocity component at the boundary plane. A simple first-order extrapolation is used to compute $k$ and $\omega$. For the inflow condition $\left(\mathbf{u}_{p h y} \cdot \mathbf{n} \geq 0\right), k_{b}=k_{\infty}$ and $\omega_{b}=\omega_{\infty}$, whereas for the outflow condition $\left(\mathbf{u}_{p h y} \cdot \mathbf{n}<0\right), k_{b}=k_{p h y}$ and $\omega_{b}=\omega_{p h y}$.

In the far-field, the flow state $\left[\mathbf{u}_{\infty}, p_{\infty}, T_{\infty}, k_{\infty}, \omega_{\infty}\right]$ is $\left[(80,0,0)^{T} \mathrm{~m} / \mathrm{s}, 101325 \mathrm{~Pa}, 298.15 \mathrm{~K}, 0,0\right]$.

\section{III.D. Starting flow condition}

The starting flow condition is obtained using a compressible laminar simulation by Grottadaurea and Rona. ${ }^{7}$ At the beginning of the computation, the inflow is changed into a turbulent boundary layer, which was developed as detailed in appendix A. At the outflow, only the back pressure $p=p_{\infty}$ is imposed.

\section{Unsteady aerodynamic predictions}

\section{IV.A. Flow instability}

From the starting flow condition, the simulation is time-marched to $26 L / u_{\infty}$ to allow a self-sustained cavity flow instability to develop. A fully developed instability is characterized by a statistically stationary flow. This condition is reached at $22.4 L / u_{\infty}$. The data presented in this section is collected after $22.4 L / u_{\infty}$ in the statistically stationary flow regime. 
The sequence of Figs. 3(a-d) shows four snapshots of the flow in the enclosure and immediate surroundings. A small portion of the full computational domain $( \pm 0.6 L, \pm 0.6 L, L)$ is visualized near the cavity. The four snapshots are a time sequence that show the interaction of convected vorticity at the top of the enclosure with the cavity downstream edge. The interaction takes place over a period $\mathcal{T}=0.7745 L / u_{\infty}$. The unsteady recirculation pattern in each snapshot is visualized by the tracing of streamlines in the short-time averaged velocity field.

The $L / D=2.5$ shallow cavity is characterized by a large recirculation in the enclosure. A shear layer spans across the cavity opening. In the shear flow, the streamlines identify the presence of a downstream convecting vortex, which is shown in Fig. $3(\mathrm{~b})$, centered at $x=0.35 L$ and $z=0.875 D$. The convecting vortex size is smaller with respect to the main recirculation inside the enclosure, centred at about $x=0.23 L$ and $z=0.5 D$. As it approaches the downstream wall in Fig. 3(b), the vortex strength appears to increase, as indicated by the packing of the streamlines. The interaction of the convected vortex with the cavity trailing edge produces pressure waves ${ }^{22}$ that radiate to the far-field where they are perceived as aerodynamic noise. The $L / D=2.5$ shallow cavity flow is found to have a dominant instability mode at $S t=1.332$ at $M_{\infty}=0.235$. The maximum reverse flow velocity inside the enclosure is $u_{1}=-37 \mathrm{~m} / \mathrm{s}$.

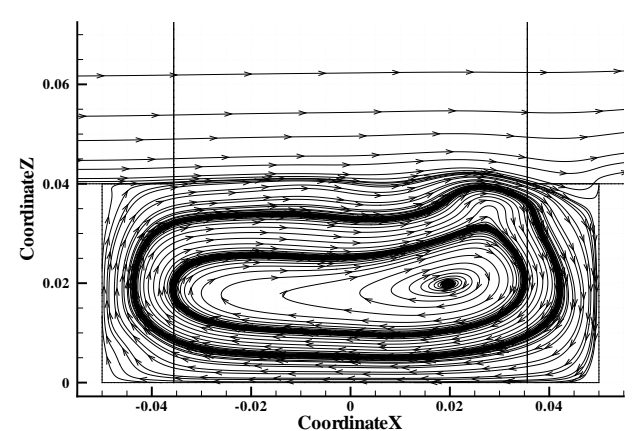

(a) $1 / 4 \mathcal{T}$

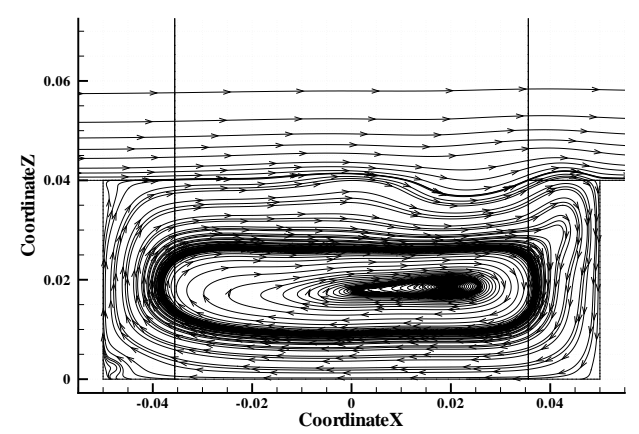

(c) $3 / 4 \mathcal{T}$

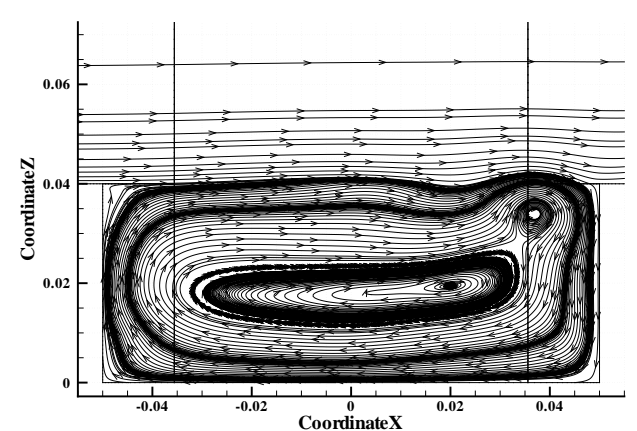

(b) $1 / 2 \mathcal{T}$

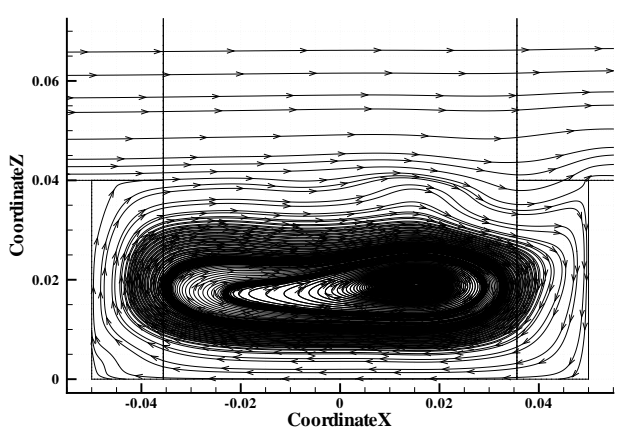

(d) $\mathcal{T}$

Figure 3. Streamlines in the short-time averaged velocity field. $y=0$ plane, $L / D=2.5$ shallow cavity.

The sequence of Figs. 4(a-d) shows short-time averaged flow snapshots from the $L / D=0.713$ deep cavity simulation, taken at increasing computational time. The velocity field is visualized by the tracing of streamlines in the short-time averaged flow. The four snapshots show a time-dependent vortex structure. The vortex structure evolves over a much longer period $\mathcal{T}=1.853$ in the $L / D=0.713$ deep cavity with respect to the $L / D=2.5$ shallow cavity. Two additional smaller recirculation zones are identified at the bottom cavity corner and just under the leading edge of the cavity, where the upstream boundary layer separates, as shown in Fig. 3(a). The dominant instability mode corresponds to $S t=0.539$ in the $L / D=0.71$ cavity. The maximum reverse flow velocity inside the enclosure is approximately $u_{1}=-51 \mathrm{~m} / \mathrm{s}$. Essentially the same azimuthal instability was predicted by an unsteady inviscid cylindrical cavity model by Rona \& Grottadaurea $^{6}$ at $S t=0.53$. 


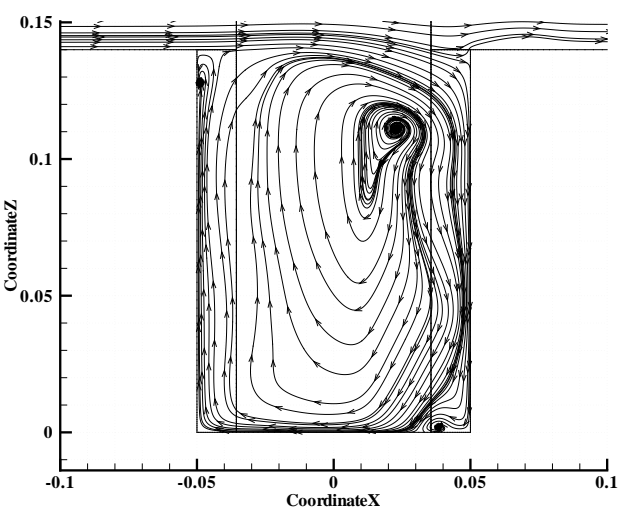

(a) $1 / 4 \mathcal{T}$

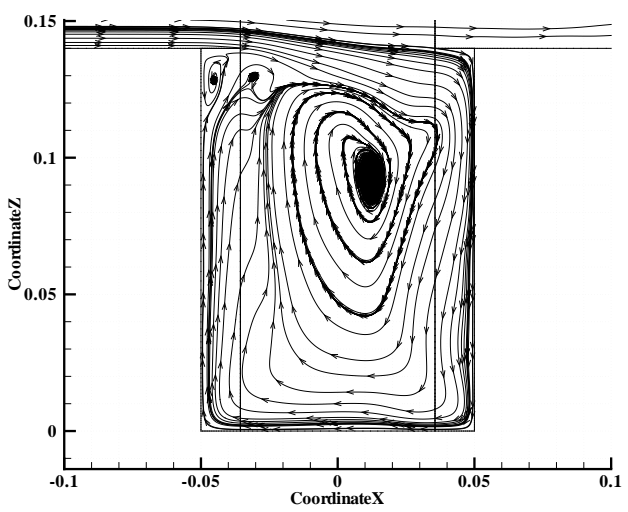

(c) $3 / 4 \mathcal{T}$

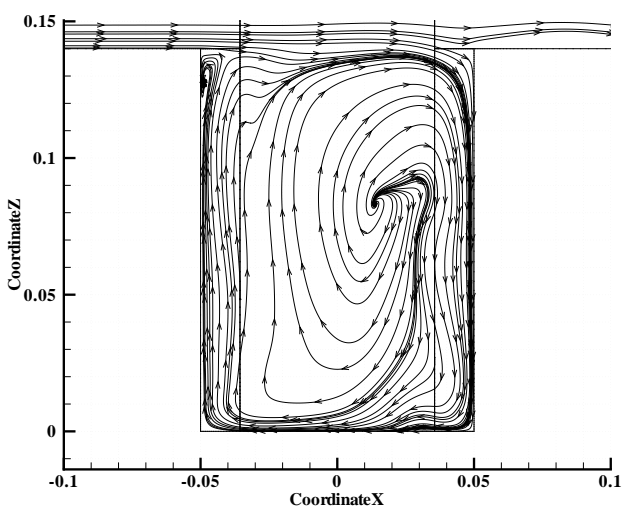

(b) $1 / 2 \mathcal{T}$

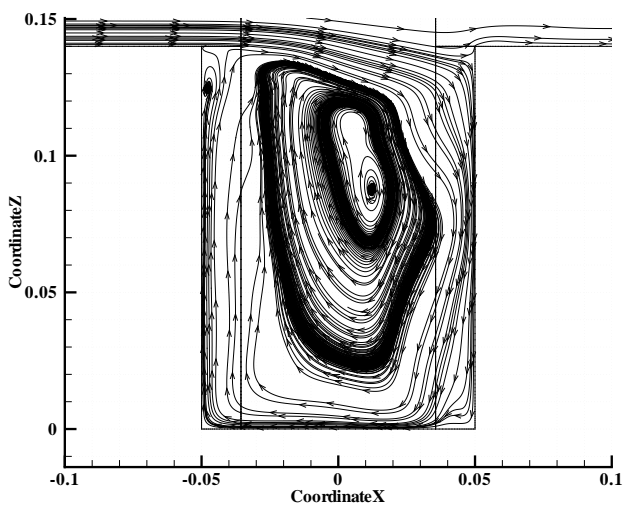

(d) $\mathcal{T}$

Figure 4. Streamlines in the short-time averaged velocity field. $y=0$ plane, $L / D=0.713$ deep cavity. 


\section{IV.B. Near-field Pressure Fluctuation}

Figs. 5(a-d) and 6(a-d) show the near-field pressure fluctuation in the $L / D=2.5$ shallow cavity and in the $L / D=0.713$ deep cavity. In Figs. $5(\mathrm{a}-\mathrm{d})$ and $6(\mathrm{a}-\mathrm{d})$ only a portion of the computational domain of extent $( \pm 10 L, \pm 10 L, 6.5 L)$ is visualized. Outside this area, the pressure waves are damped by the rapid mesh stretching. Contours of pressure fluctuation, $p^{\prime}=p-p_{\infty}$ are shown by ten solid lines $\left(p^{\prime} \geq 0\right)$ and nine dashed lines $\left(p^{\prime}<0\right)$. The contour spacing $\Delta p^{\prime}=2 \mathrm{~Pa}$ in the $L / D=2.5$ shallow cavity and $\Delta p=5 \mathrm{~Pa}$ in the $L / D=0.713$ deep cavity.

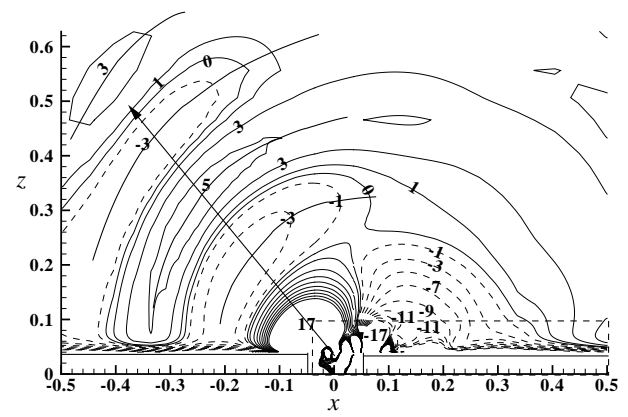

(a) $1 / 4 \mathcal{T}$

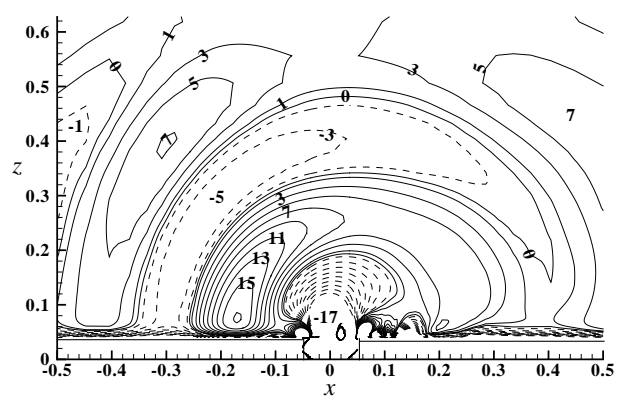

(c) $3 / 4 \mathcal{T}$

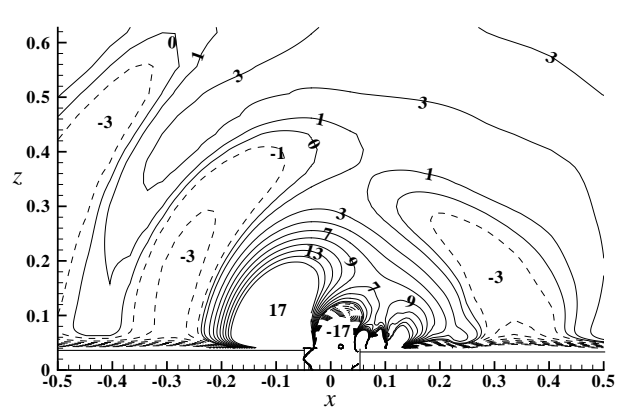

(b) $1 / 2 \mathcal{T}$

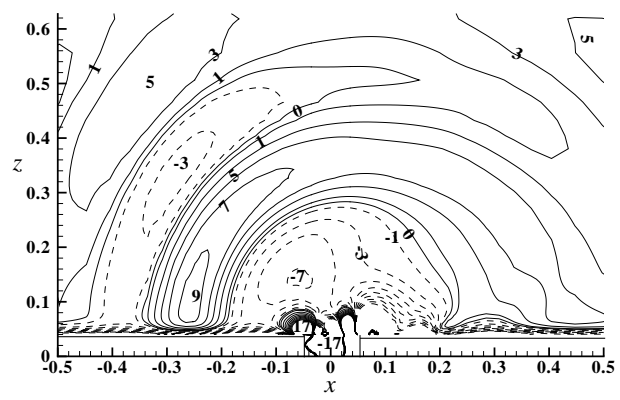

(d) $\mathcal{T}$

Figure 5. Pressure fluctuations iso-contours in Pa. $y=0$ plane, $L / D=2.5$ shallow cavity.

In figures 5(a) and 6(a) the time-dependent pressure fluctuation is characterized by small structures located inside the enclosure and just above the downstream bulkhead. These fluctuations are hydrodynamic in nature and are associated to the generation of vorticity in the shear layer spanning the cavity opening. This vorticity is injected and ejected alternatively at the cavity trailing edge and the ejected structures convect over the downstream bulkhead. The pressure fluctuation associated to these vorticity clusters results in the compact small-scale contours of Figs. 5(a) and 6(a) along the cavity rear bulkhead. The region where hydrodynamic pressure fluctuations are dominant is highlighted in Figs. 5(a) and 6(a) by a dashed line rectangle.

Outside this region, the contours of pressure fluctuation show larger structures. The time sequence of figures $5(\mathrm{a}-\mathrm{d})$ and $6(\mathrm{a}-\mathrm{d})$ show these structures radiating away from the enclosure. The fluctuations of pressure in this area are due to the cavity acoustic radiation and the contours here describe the acoustic near-field. In figure 5(a) an acoustic wavefront is shown propagating in the upstream direction. The position of this wavefront is marked by a continuous thick arc. This suggests that in the $L / D=2.5$ cavity the main radiation is directed upstream.

In figure 6(a), two acoustic fronts are identified in the near-field of the $L / D=0.713$ deep cavity. There is a dominant upstream radiation, just like in the $L / D=2.5$ cavity, that is accompanied by a downstream pressure front, which is peculiar to the $L / D=0.713$ deep cavity.

The pressure field associated to the $L / D=2.5$ shallow cavity is characterized by a lower amplitude fluctuation with respect to the $L / D=0.71$ deep cavity, consistently with the previous inviscid simulations. ${ }^{6}$ Specifically, the highest positive contour away from the cavity where the near-acoustic radiation dominates 


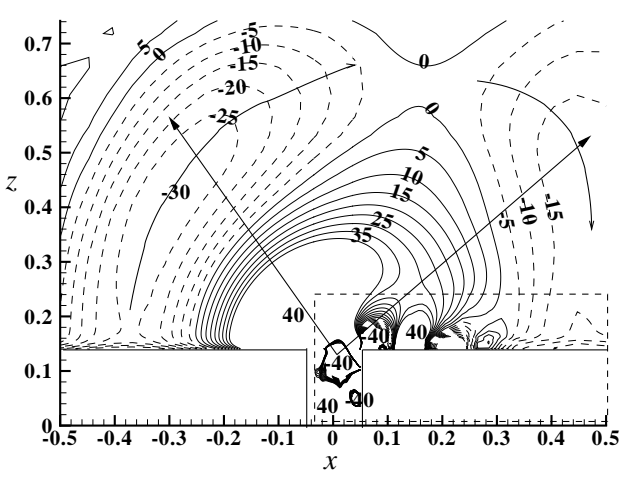

(a) $1 / 4 \mathcal{T}$

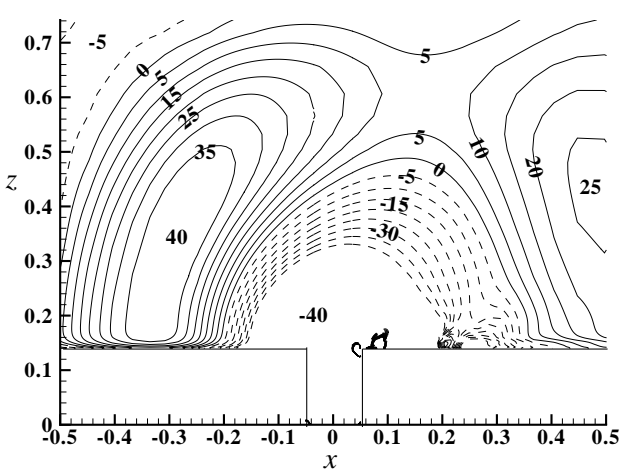

(c) $3 / 4 \mathcal{T}$

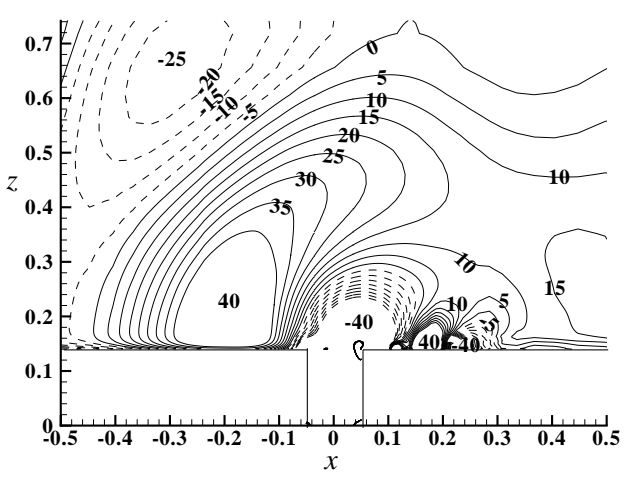

(b) $1 / 2 \mathcal{T}$

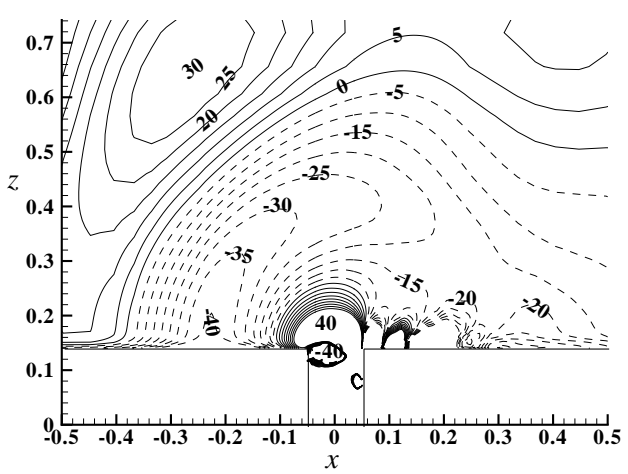

(d) $\mathcal{T}$

Figure 6. Pressure fluctuations iso-contours in Pa. $y=0$ plane, $L / D=0.713$ deep cavity. 
is $15 \mathrm{~Pa}$, as shown in figure 5(c). The largest positive acoustic near-field pressure fluctuation contour in the $L / D=0.71$ predictions is $40 \mathrm{~Pa}$, as shown in figure 6(c).

\section{IV.C. Near-field Sound Pressure Level}

The near-field Sound Pressure Level (SPL) has been estimated by averaging the time-resolved pressure predictions over one fundamental period of cavity flow instability $\mathcal{T}$. $N=43$ frames are used from the $L / D=2.5$ shallow cavity simulation and $N=72$ frames from the $L / D=0.71$ deep cavity simulation.

$$
\begin{aligned}
S P L & =20 \log \left(p_{\text {rms }} / p_{0}\right) \\
p_{\text {rms }} & =\sqrt{\frac{1}{N} \sum_{1}^{N}(p-\bar{p})^{2}}
\end{aligned}
$$

In equation (11), the reference pressure is $p_{0}=20 \mu \mathrm{Pa}, p_{r m s}$ is the root mean square pressure fluctuation and $\bar{p}=1 / N \sum_{1}^{N} p$ is the mean pressure of the $N$ frames.

Figures $8(\mathrm{a}-\mathrm{b})$ and $7(\mathrm{a}-\mathrm{b})$ show SPL iso-contours in the $L / D=2.5$ shallow cavity and in the $L / D=0.713$ deep cavity respectively. The contour spacing is $\triangle S P L=5 d B$. Figures 7 (a) and $8($ a) show the SPL isocontours in the $\phi=0^{\circ}$ plane, and figures 7(b) and 8(b) show the SPL iso-contours in the $\phi=90^{\circ}$ plane.

The $L / D=2.5$ cavity shows the main acoustic radiation being in the direction of $\left(\psi=126^{\circ}, \phi=0^{\circ}\right)$, as shown in figure $7(\mathrm{a})$. In the spanwise plane, the predicted sound wave is asymmetric with respect to the cavity mid-span, with a stronger radiation at the azimuthal angle $\psi=60^{\circ}$ at $\phi=90^{\circ}$, as shown in figure $7(\mathrm{~b})$.

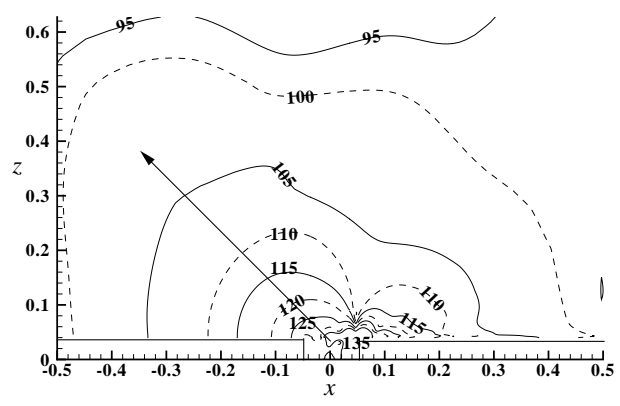

(a) $\phi=0^{\circ}$

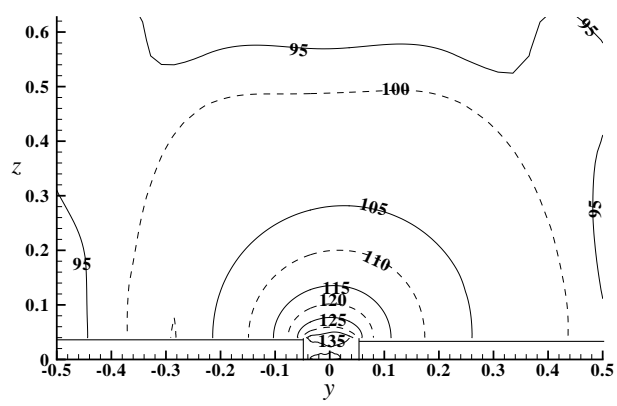

(b) $\phi=90^{\circ}$

Figure 7. Near-field SPL $[d B] L / D=2.5$ shallow cavity.

At the same radial distance, the deep cavity acoustic near-field is about $15 \mathrm{~dB}$ re $20 \mu \mathrm{Pa}$ louder with respect to the shallow cavity. For instance, the $100 \mathrm{~dB}$ contour in figure 7 (b) covers about the same position as the $115 \mathrm{~dB}$ contour in figure 8(b). As noted in the near-field pressure fluctuation contours of figure 6(a), the deep cavity has two preferential directivity directions for the near-field acoustic radiation. The SPL contours of figure 8 (a) enable to estimate the directions of preferential radiation in the $\phi=0^{\circ}$ plane. These are at an azimuthal angle $\psi=114^{\circ}$ in the upstream direction and at $\psi=30^{\circ}$ in the downstream direction. Figure 8 (b) shows the directivity of the deep cavity in the spanwise plane, at $\phi=90^{\circ}$. The contours appear to be more circular and symmetric with respect to figure $7(\mathrm{~b})$.

An easier comparison of the near-field acoustic radiation directivity is provided by figure 9 (a-b), where the predicted SPL from both $L / D=0.713$ and $L / D=2.5$ are shown at the same radial distance $r=5 L$. The $L / D=0.713$ cavity is confirmed to be the louder flow, with two maxima of $S P L=121 \mathrm{~dB}$ at $\left(\psi=114^{\circ}, \phi=0^{\circ}\right)$ and $S P L=118.5 \mathrm{~dB}$ at $\left(\psi=60^{\circ}, \phi=0^{\circ}\right)$, shown in figure $9(\mathrm{~b})$. A lower directivity maximum of $S P L=104 \mathrm{~dB}$ is predicted at $\left(\psi=126^{\circ}, \phi=0^{\circ}\right)$ in the $L / D=2.5$ cavity. 


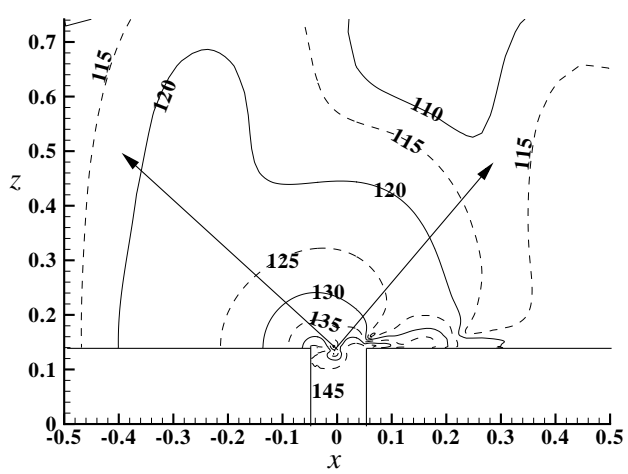

(a) $\phi=0^{\circ}$

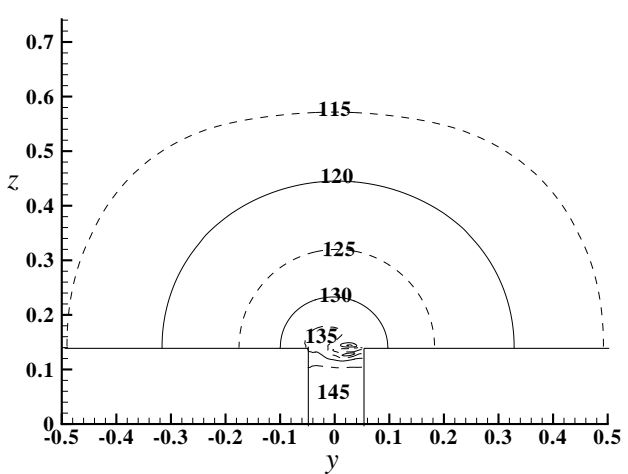

(b) $\phi=90^{\circ}$

Figure 8. Near-field SPL $[d B] L / D=0.713$ deep cavity.

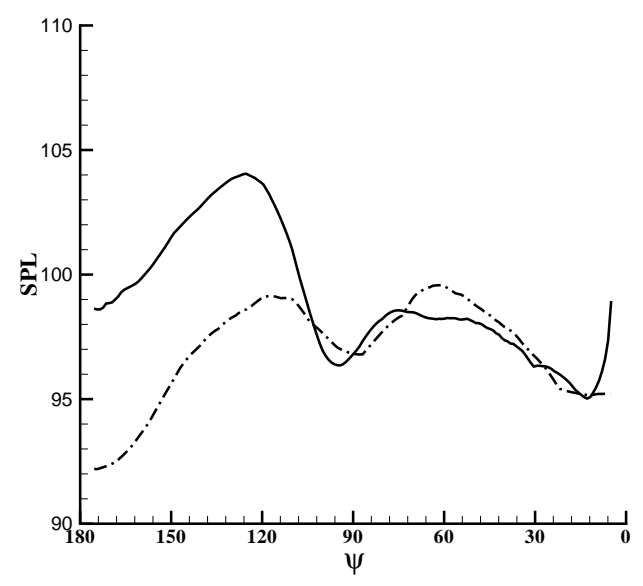

(a) $L / D=2.5$ shallow cavity

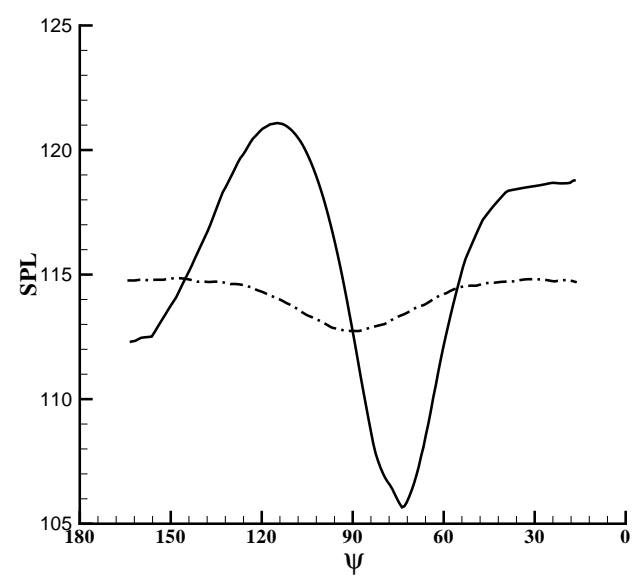

(b) $L / D=0.713$ deep cavity

Figure 9. SPL at $r=$ const $=5 L$ above cavity opening, $\phi=0^{\circ}[-]$ and $\phi=90^{\circ}[-\cdot-]$ 


\section{Concluding remarks}

This paper addresses the fluid dynamic instabilities and the radiating pressure that develop in a cylindrical subsonic turbulent cavity flow. The flow instability is peculiar to this geometry and the effects of the incoming boundary layer velocity profile as well as of the small scale mixing on the unsteady flow pattern and near-field noise were investigated.

Time-dependent Detached Eddy Simulations of cylindrical cavity flows at two aspect ratio highlighted interesting features of both the flow field and the radiating pressure pattern.

The time dependent predictions of the radiating pressure field in both shallow and deep cavity configurations indicate a significant noise radiation from these flows. The acoustic near-field is not symmetric with respect to the cavity mid-span. This asymmetry is most likely linked to the azimuthal instability modes that develop in the enclosure. The mode selection in the 'hydrodynamic' flow region that spans the cavity opening drives the production of sound along preferential directions in the acoustic near-field. The persistance of such asymmetric radiation in the acoustic far-field is likely to be significant for the noise performance of cylindrical aircraft fuel vents at landing.

The directivity has been investigated at a costant radial distance in streamwise and spanwise planes. The two configurations herein investigated are characterized by a similar upstream main directivity. The $L / D=0.713$ cavity is louder and displays a secondary downstream peak. In the spanwise plane, the acoustic wave from the $L / D=2.5$ cavity is asymmetric whereas it is symmetric in the $L / D=0.713$ cavity.

Given the potential impact of asymmetric cavity instability modes on airframe noise, further research is required to determine the drivers behind mode staging, together with a better assessment of the effects such modes have on the sound perceived in the acoustic far-field. This will benefit research on airframe landing noise reduction by tackling the fuel vent noise source.

\section{Acknowledgments}

This research project has been supported by a Marie Curie Early Stage Research Training Fellowship of the European Community's Sixth Framework Programme under contract number MEST CT 2005020301.

This work was carried out under the HPC-EUROPA project (RII3-CT-2003-506079) with the support of the European Community - Research Infrastructure Action under FP6 "Structuring the European Research Area" Programme, using the supercomputer facility at Cineca, Bologna, Italy.

This work made use of the facilities of HECToR, the UK's national high-performance computing service, which is provided by the UoE HPCx Ltd at the University of Edinburgh, Cray Inc and NAG Ltd, and funded by the Office of Science and Technology through EPSRC's High End Computing Programme.

\section{References}

${ }^{1}$ Wilcox, D., Turbulence modeling for CFD, D C W Industries, 2nd ed., December 2002.

${ }^{2}$ Yoshizawa, A., "Statistical theory for compressible shear flows with the application of subgrid modelling," Physics of Fluids A, Vol. 29, 1986, pp. 2152-63.

${ }^{3}$ Bunyakin, A. V., "Laminar boundary layer in a flow past an aerofoil with a circular cavity," Fluid Dynamics, Vol. 33, No. 2, 1998, pp. $196-200$

${ }^{4}$ Hering, T., Dybenko, J., and Savory, E., "Experimental verification of CFD modeling of turbulent flow over circular cavities," Canadian Society of Mechanical Engineering Forum, Kananaskis, Canada, May 2006.

${ }^{5}$ Dybenko, J., Hering, T., and Savory, E., "Turbulent flow over circular cylindrical cavities with varying depth to diameter ratio," International Council of the Aeronautical Sciences Congress, Hamburg, Germany, September 2006.

${ }^{6}$ Grottadaurea, M. and Rona, A., "Noise sources from a cylindrical cavity," 13th AIAA/CEAS Aeroacoustics Conference, edited by AIAA, No. 2007-3723, Rome, Italy, May 2007.

${ }^{7}$ Grottadaurea, M. and Rona, A., "The role of the inflow momentum thickness in subsonic cylindrical cavity noise generation," 14 th International Congress on Sound and Vibration, No. 165, Cairns, Australia, July 2007.

${ }^{8}$ Rossiter, J., "Wind-Tunnel Experiments on the Flow over Rectangular Cavities at Subsonic and Transonic Speeds," Technical Report 3438, Aeronautical Research Council Reports and Memoranda, 1964.

${ }^{9}$ Rona, A., "The acoustic resonance of rectangular and cylindrical cavities," J. Algorithms \&3 Computational Technology, Vol. 1, No. 3, 2007, pp. 329-55.

${ }^{10}$ Colonius, T. and Lele, S., "Computational aeroacoustics: progress on nonlinear problems of sound generation," Progress on Aerospace Sciences, Vol. 40, 2004, pp. 365-416.

${ }^{11}$ Rowley, C., Colonius, T., and Basu, A., "On Self-sustained Oscillations in Two-dimensional Compressible Flow Over Rectangular Cavities," J. Fluid Mechanics, Vol. 455, 2002, pp. 315-46. 
${ }^{12}$ Tam, C., "Computational Aeroacoustics: an Overview of Computational Challenges and Applications," Int. J. Computational Fluid Dynamics, Vol. 18, No. 6, 2004, pp. 547-67.

${ }^{13}$ Charwat, A., Roos, J., Dewey, F., and Hitz, J., "An investigation of separated flows. Part I: The pressure field," J. Aerospace Sci., Vol. 28, No. 6, 1961, pp. 457-70.

${ }^{14}$ Colonius, T., Basu, A. J., and Rowley, C. W., "Numerical investigation of the flow past a cavity," 5th AIAA/CEAS Aeroacoustics Conference, No. 1912 in AIAA, Greater Settle, Washington, 1999.

${ }^{15}$ Colonius, T., "An overview of simulation, modeling, and active control of flow/acoustic resonance in open cavities," Progress on Aerospace Sciences, Vol. 40, 2001, pp. 365-416.

${ }^{16}$ Chen-Chuan Fan, T., Hybrid Reynolds-Averaged / Large-Eddy Simulations of Ramped-Cavity and Compression Ramp Flow-fields, Degree of master of science, Department of Mechanical and Aerospace Engineering, Raleigh, North Carolina, July 2002.

${ }^{17}$ Menter, F., "Improved Two Equation $k-\omega$ Turbulence Models for Aerodynamic Flows," Technical Memorandum 103975 , NASA Ames Research Center, California, October 1992.

${ }^{18}$ Manna, M., A three dimensional high resolution compressible flow solver, Phd thesis, faculty of applied science, Université Catholique de Louvain, October 1992.

${ }^{19}$ Rona, A. and Brooksbank, E., "Injection parameters for an effective passive control of the cavity flow instability," 40th AIAA Aerospace Sciences Meeting \& Exhibit, edited by AIAA, Reno, NV, January 2002.

${ }^{20}$ Giles, M., "Non-reflecting boundary conditions for Euler Equation calculation," AIAA Journal, Vol. 28, No. 12, 1990, pp. 2050-58.

${ }^{21}$ AIAA, "Recommended Practice. The CFD General Notation System. Standard Interface Data Structures," AIAA R 101-2002, AIAA, Reston, VA, USA, 2002.

${ }^{22}$ Powell, A., "Theory of vortex sound," J. Acoustical Society of America, Vol. 36, No. 1, 1964, pp. 177-95.

${ }^{23}$ Pengyuan, Y. and Biondini, L., "Turbulent boundary layer characterization at the ENEA wind tunnel," Technical report, Università degli studi of Roma Tre, 2007.

${ }^{24}$ De Graaff, D. and Eaton, J., "Reynolds-number scaling of the flat-plate turbulent boundary layer," J. Fluid Mechanics, Vol. 422, June 2000, pp. 319-46.

${ }^{25}$ Marusic, I. and Kunkel, G., "Streamwise turbulence intensity formulation for flat-plate boundary layer," Physics of Fluids, Vol. 15, No. 8, August 2003, pp. 2461-64.

${ }^{26}$ White, F., Viscous fluid flow, McGraw-Hill Series in Mechanical Engineering, 2nd ed., 1991.

\section{A. Turbulent inflow boundary layer}

The turbulence intensity in the outer region of a fully developed turbulent boundary layer is described by the empirical function $f_{3}$ at $z^{+}>150$. The latter is obtained by the interpolation of experimental data from the ENEA/DIMI wind tunnel. ${ }^{23}$ Figure 10 shows the normalized turbulence intensity $u^{\prime 2+}=u^{\prime 2} / u_{\tau}^{2}$ versus the normalized wall distance $z^{+}=z u_{\tau} / \nu$. The data from the ENEA wind tunnel is plotted with the symbols $\diamond, \square, \triangle$ and $\circ$ over the range $67 \leq z^{+} \leq 5000$. The experimental predictions from De Graaff and Eaton $^{24}$ are also shown, covering the wider range $4 \leq z^{+} \leq 9000$. Over the common range, the two data sets show a good agreement, with the spread among data sets being due to the variation in $R e_{\theta}$. In the Ente Nazionale per l'Energia e l'Ambiente (Italian national research center for Energy and Environment) measurement, the outer boundary layer was traversed, therefore a model for the inner region is used in the simulation. Marusic and Kunkel ${ }^{25}$ proposed an empirical formula $\left(f_{1}\right)$ to evaluate the turbulence intensity in the inner region as a function of $R_{\theta}$ and $z^{+}$.

\begin{tabular}{|c|c|c|c|}
\hline$\#$ & Symbol & $R e_{\theta}$ & Author \\
\hline 1 & $\diamond$ & 4900 & ENEA \\
\hline 2 & $\circ$ & 6050 & ENEA \\
\hline 3 & $\square$ & 8250 & ENEA \\
\hline 4 & $\triangle$ & 8760 & ENEA \\
\hline 5 & $\times$ & 5200 & De Graaff-Eaton \\
\hline 6 & + & 13000 & De Graaff-Eaton \\
\hline 7 & $*$ & 31000 & De Graaff-Eaton \\
\hline$f_{1}$ & $-\cdot-$ & 5200 & Marusic-Kunkel \\
\hline$f_{3}$ & - & 8760 & ENEA \\
\hline
\end{tabular}

Table 2. Summary table. 


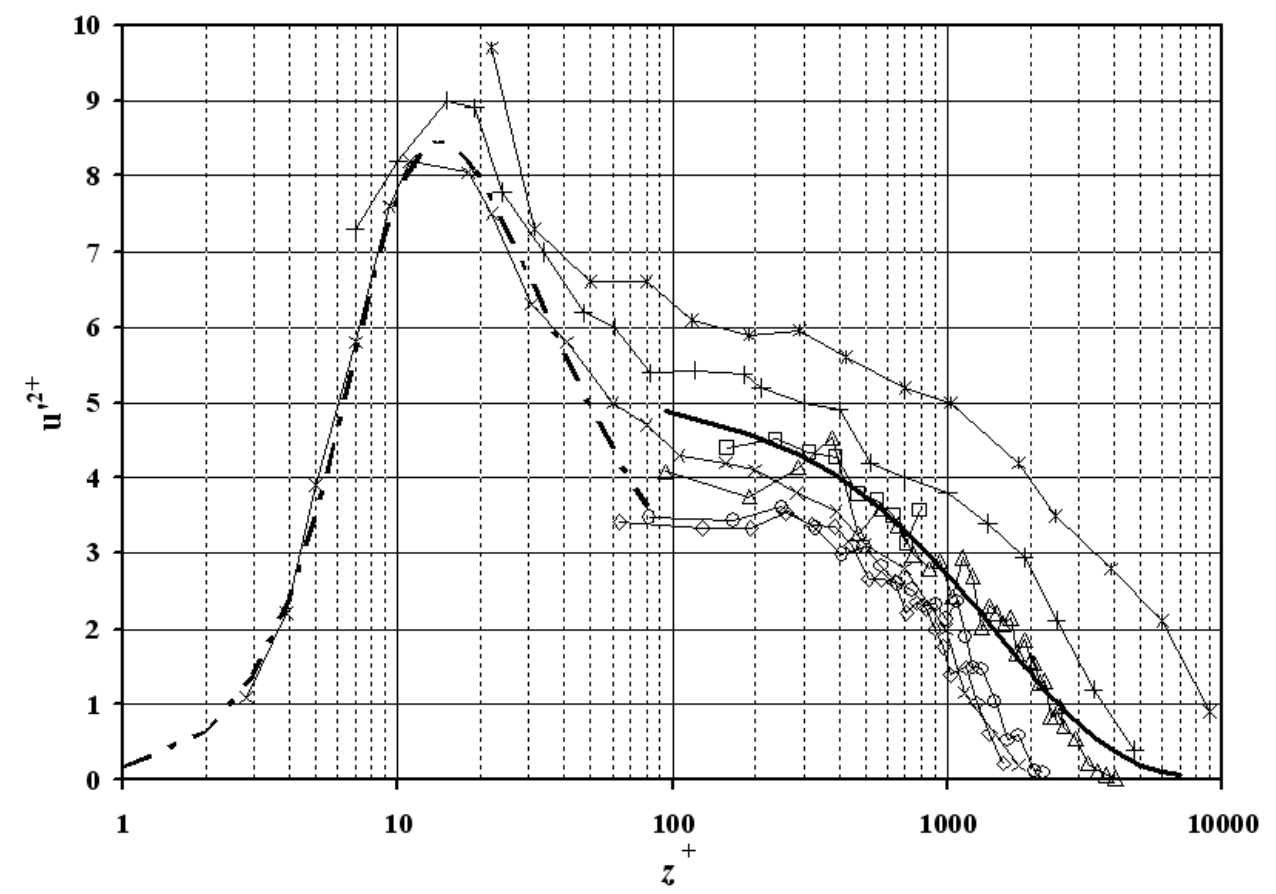

Figure 10. Normalized turbulent intensity. Symbols and conditions are given in table 2 .

$u^{\prime 2+}$ is analytically described by:

$$
u^{\prime 2+}= \begin{cases}f_{1}\left(z^{+}, R e_{\theta}\right), & z^{+} \leq z_{\text {inner }}^{+} \\ f_{2}\left(z^{+}, R e_{\theta}\right), & z_{\text {inner }}^{+}<z^{+}<z_{\text {outer }}^{+} \\ f_{3}\left(z^{+}, R e_{\theta}\right), & z^{+} \geq z_{\text {outer }}^{+}\end{cases}
$$

$f_{2}$ is a function of $R e_{\theta}$ and $z^{+}$and it is obtained as a gradient-matched cubic curve-fit as proposed by Marusic and $\mathrm{Kunkel}^{25}$ for simplicity.

The turbulent kinetic energy and its specific dissipation rate are defined as:

$$
\begin{aligned}
k & =\frac{3}{2} u^{\prime 2+} u_{\tau}^{2} \\
\omega & =\frac{\rho k}{\mu_{t}}
\end{aligned}
$$

At the inflow, $\rho=\rho_{\infty}=1.225 \mathrm{~kg} / \mathrm{m}^{3}$ and $\mathbf{u}$ is obtained from the interpolation of the ENEA/DIMI boundary layer data at $z^{+} \geq 100$ and is modelled using Spalding's law of the wall for a zero pressure gradient boundary layer at $z^{+}<100 .{ }^{26} k$ and $\omega$ are obtaining by equation (13) using equation (12). 